\section{A suplementação de L-carnitina não promove alterações na taxa metabólica de repouso e na utilização dos substratos energéticos em indivíduos ativos}

\author{
The supplementation of L-carnitine does not promote \\ alterations in the resting metabolic rate and in the use of \\ energetic substrates in physically active individuals
}

Christianne de Faria Coelho', João Felipe Mota²,

Fabrício César de Paula Ravagnani ${ }^{3}$, Roberto Carlos Burini ${ }^{4}$

\section{RESUMO}

Objetivo: Avaliar o efeito da suplementação de L-carnitina, por 30 dias, sobre a taxa metabólica de repouso (TMR) e oxidação de ácidos graxos livres (AGL), em repouso e exercício. Sujeitos e Métodos: Vinte e um voluntários ativos (40 a 58 anos) com sobrepeso foram randomizados em dois grupos: suplementado (GS; $N=11 ; 1,8 \mathrm{~g} /$ dia de L-carnitina) e placebo (GP; $N=10$; maltodextrina). Foi feita avaliação da ingestão calórica, antropometria, determinação daTMR, $\mathrm{VO}_{2 \text { máx' }}$ quociente respiratório e AGL plasmáticos. Resultados: Não houve diferença significativa na ingestão $(-244,66$ vs. $-126,00 \mathrm{kcal} / \mathrm{dia})$, composição corporal $\left(-0,07\right.$ vs. $\left.-0,17 \mathrm{~kg} / \mathrm{m}^{2}\right), \mathrm{TMR}(0,06 \mathrm{vs} .-0,02 \mathrm{kcal} /$ dia), quociente respiratório em repouso $(3,69$ vs. $-1,01)$ e exercício $(0,01$ vs. $-0,01)$ e $\mathrm{VO}_{2 \text { máx }}(0,50$ vs. $1,25 \mathrm{~mL} / \mathrm{kg} / \mathrm{min}$ ) para o grupo GS em relação ao GP. Houve aumento dos AGL em repouso no GP $(0,27)$, porém sem diferenças no exercício para os grupos. Conclusão: Não houve efeito da L-carnitina em nenhuma das variáveis analisadas no estudo. Arq Bras Endocrinol Metab. 2010;54(1):37-44

\section{Descritores}

Exercício físico; suplementação; L-carnitina; composição corporal; ácidos graxos livres

\begin{abstract}
Purpose: To investigate the effects of L-carnitine supplementation, over thirty days, on the resting metabolic rate (RMR) and oxidation of free fatty acids (FFA) under rested or exercised conditions. Subjects and methods: Twenty-one overweight active volunteers (40 to 58 years old) were randomized into two groups: supplemented (GS; $\mathrm{N}=11 ; 1,8 \mathrm{~g} / \mathrm{day}$ of L-carnitine) or placebo (GP; $\mathrm{N}=10$; maltodextrin). Caloric intake, anthropometry, $\mathrm{RMR}, \mathrm{VO}_{2 \max ^{\prime}}$ respiratory exchange ratio and plasma FFA were measured. Results: No significant changes were found in the caloric intake (-244,66 vs. $-126,00 \mathrm{kcal} / \mathrm{day})$, body composition $\left(-0.07 \mathrm{vs} .-0.17 \mathrm{~kg} / \mathrm{m}^{2}\right)$, RMR $(0.06$ vs. $-0.02 \mathrm{kcal} / \mathrm{day})$, respiratory exchange ratio at rest $(3.69 \mathrm{vs}$. -1.01$)$ and exercise $(0.01 \mathrm{vs} .-0.01)$ or VO $_{2 \max }(0.50$ vs. $1.25 \mathrm{~mL} / \mathrm{kg} / \mathrm{min}$ ) between GS and GP. Plasma FFA levels were increased under resting conditions only in the GP group (0.27), but no significant changes were observed before or after physical activity in any of the groups. Conclusion: Supplementation with L-carnitine caused no changes in the variables analyzed in this study. Arq Bras Endocrinol Metab. 2010;54(1):37-44
\end{abstract}

Keywords

Exercise; supplementation; L-carnitine; body composition; free fatty acids
${ }^{1}$ Programa de Pós-graduação em Nutrição Humana Aplicada (Pronut), Universidade de São Paulo (USP). Universidade para o Desenvolvimento do Estado e da Região do Pantanal (Uniderp), Campo Grande, MS, Brasil

2 Departamento de Fisiologia da Nutrição, Escola Paulista de Medicina, Universidade Federal de São Paulo (Unifesp/EPM).

Departamento de Nutrição, Universidade São Francisco, Bragança Paulista, SP, Brasil ${ }^{3}$ Universidade Federal do Mato Grosso do Sul (UFMS). Universidade Católica Dom Bosco (UCDB), Campo Grande, MS, Brasil ${ }^{4}$ Centro de Metabolismo em Exercício e Nutrição (CeMENutri), Departamento de Saúde Pública, Faculdade de Medicina de Botucatu, Universidade Estadual Paulista (Unesp)

Correspondência para: Christianne de Faria Coelho Rua Garcia Neto, 395 78065-050 - Cuiabá, MT, Brasil christianne.coelho@hotmail.com

Recebido em 16/Abr/2009 Aceito em 30/Out/2009 


\section{INTRODUÇÃO}

$\mathrm{O}$ uso de suplementos nutricionais tem crescido ao longo das últimas décadas. Atletas e indivíduos fisicamente ativos acreditam no potencial ergogênico de diversas substâncias, sobretudo para a melhoria do desempenho físico e/ou estética corporal (1).

Entre as substâncias que têm recebido grande atenção de pesquisadores, técnicos, atletas e demais indivíduos, destaca-se a carnitina. Por volta de 1955, a L-carnitina foi relacionada à oxidação de ácidos graxos (2) e, a partir da década de 1980, sua suplementação passou a ser recomendada para o tratamento de doenças associadas às concentrações diminuídas desta amina quartenária (3).

Atualmente, a carnitina tem sido frequentemente utilizada por indivíduos ativos como coadjuvante na redução de gordura corporal, sendo usada comercialmente nos suplementos denominados termogênicos (1).

Os possíveis efeitos termogênico e emagrecedor da substância baseiam-se no fato de que a L-carnitina atua nas reações de transferência dos ácidos graxos cadeia longa do citosol para a mitocôndria, facilitando a oxidação desses e a consequente geração de ATP (4-5).

Assim, a hipótese proposta é de que a administração de carnitina poderia ter efeito, tanto no repouso quanto no exercício, sobre a utilização dos ácidos graxos livres pelo músculo, mudando dessa maneira a oxidação de substratos de carboidratos para lipídios, reduzindo o valor do quociente respiratório (QR) (2).

Especificamente em relação ao exercício físico, a suplementação de L-carnitina poderia elevar os seus níveis musculares, aumentando assim a liberação de CoA livre e reduzindo a razão acetil-CoA/CoA livre nas mitocôndrias. A menor relação acetil-CoA/CoA estimularia a atividade da piruvato desidrogenase e aumentaria, dessa forma, o fluxo de substratos através do ciclo de Krebs e, como consequência, o $\mathrm{VO}_{2 \text { máx }}(6)$.

No entanto, todos os efeitos citados acima ainda são controversos, particularmente nos indivíduos ativos de idades mais avançadas. Logo, este trabalho tem como objetivo avaliar o efeito da suplementação de L-carnitina por quatro semanas sobre a taxa metabólica de repouso e a oxidação de ácidos graxos livres no estado de repouso e durante a prática de exercício físico aeróbico em indivíduos ativos com excesso de peso e obesidade.

\section{SUJEITOS E MÉTODOS}

Foram selecionados 30 indivíduos, acima de 40 anos (46 \pm 5 anos), com diagnóstico de excesso de peso e obesidade (7), participantes há 12 semanas do programa de extensão universitária com exercícios físicos supervisionados e aconselhamento alimentar conduzidos pelo Centro de Metabolismo em Exercício e Nutrição (CeMENutri) da Faculdade de Medicina da Universidade Estadual Paulista (Unesp) de Botucatu.

Os critérios de exclusão foram: indivíduos vegetarianos, mulheres com ciclos menstruais irregulares, portadores de doenças renais, digestivas e metabólicas, que faziam uso de hormônios ou similares e drogas que interferissem no metabolismo normal, além de corticoides, hipolipemiantes como estatinas e fibratos, diuréticos e suplementos vitamínicos e/ou minerálicos.

$\mathrm{O}$ estudo cego e randomizado foi conduzido dentro dos padrões exigidos pela Declaração de Helsinki (1975), modificada em 1983 e aprovada pela Comissão de Ética em Pesquisa da Faculdade de Medicina de Botucatu, Brasil ( $n^{\circ} 112$ /protocolo 209/2003). Todos os indivíduos assinaram o termo de consentimento livre e esclarecido informando sobre a proposta e os procedimentos do programa.

O protocolo de exercícios físicos do projeto de extensão universitária foi constituído por exercícios aeróbicos, de resistência muscular localizada e flexibilidade, aplicados em cinco sessões semanais, em que o progra$\mathrm{ma} A$ foi realizado às segundas, quartas e sextas-feiras $\mathrm{e}$ o programa $\mathrm{B}$, às terças e quintas-feiras, como se segue:

Programa A: 10 minutos de alongamento e aquecimento articular; 40 minutos de caminhada (70\% a $80 \%$ da frequência cardíaca máxima); 20 minutos de flexibilidade e equilíbrio; 10 minutos de relaxamento.

Programa B: 10 minutos de alongamento e aquecimento articular; 30 minutos de resistência muscular localizada; 30 minutos de caminhada $(70 \%$ a $80 \%$ da frequência cardíaca máxima); 10 minutos de relaxamento.

Após 12 semanas de participação no projeto, os indivíduos foram randomizados em dois grupos, contendo cada grupo 7 homens e 8 mulheres. Ambos os grupos permaneceram seguindo o protocolo de exercícios físicos por mais quatro semanas acrescido de suplementação por via oral. O grupo suplementado (GS) foi composto por indivíduos que receberam cápsulas de L-carnitina em doses diárias de $1,8 \mathrm{~g}$, fracionadas nos períodos manhã e tarde ou noite, em intervalo médio de oito horas, sendo um deles uma hora antes da prática de exercícios físicos aeróbicos. O grupo placebo (GP) foi composto por indivíduos que receberam cápsulas de composto de amido com a mesma quantidade e características organolépticas. Os grupos desconheciam o su- 
plemento que estavam recebendo e todos os indivíduos foram orientados a ingerir as cápsulas ( 6 cápsulas/dia) com água ou suco de frutas. A dosagem e o período de suplementação seguiram o protocolo utilizado em outros estudos, sendo considerado seguro (8).

Ambos os grupos foram submetidos a avaliações no início (M0) e final do experimento (Ml). Entre M0 e $\mathrm{Ml}$ os sujeitos foram pesados e consultados em relação à adesão ao protocolo de exercícios e suplementação, bem como à ocorrência de efeitos adversos durante o experimento.

No primeiro dia de teste e ao final do período experimental (últimos testes), os indivíduos foram submetidos à anamnese nutricional. Os participantes foram orientados a preencher registro dietético de três dias (dois dias na semana e um no domingo), com a finalidade de se conhecer a ingestão calórica (Virtual Nutri, versão 1.0) e verificar possíveis alterações alimentares no decorrer do experimento. A dieta deveria permanecer sem alterações nas quatro semanas de suplementação. Durante todo o experimento, a ingestão de água foi estimulada para adequada hidratação, pois foi constatado baixo consumo entre os indivíduos. O estímulo ocorreu por meio de orientações específicas sobre a importância e apresentação de estratégias de mensuração da ingestão, como a utilização de garrafas com registro de volume.

Foram tomadas medidas de peso corporal e estatura para o cálculo do índice de massa corpórea (IMC) e posterior classificação dos indivíduos (7). A circunferência abdominal foi medida com fita métrica inextensível e inelástica e analisada de acordo com o sexo (9). Para estimar os valores de gordura percentual, foi utilizado o teste de impedância bioelétrica e a fórmula proposta por Gray e cols. (10).

As concentrações de ácidos graxos livres (AGL) foram dosadas no $\mathrm{M} 0$ e $\mathrm{Ml}$, pela manhã, em repouso e após jejum de 12 horas e antes e após a realização do exercício físico em esteira rolante, durante 30 minutos, a $60 \%$ do consumo máximo de oxigênio $\left(\mathrm{VO}_{2 \text { máx }}\right)$. No período anterior ao exercício, a coleta sanguínea foi realizada após 1 hora da ingestão de uma refeição padrão, contendo aproximadamente $330 \mathrm{kcal}$ (69\% de carboidratos; $13 \%$ de proteínas e $17 \%$ de lipídios). Anteriormente a essa refeição, os indivíduos estavam em jejum de 4 horas. Para a determinação das concentrações de AGL, foi utilizado o método proposto por Novak (11).

Para avaliação do consumo máximo de oxigênio $\left(\mathrm{VO}_{2 \max }\right)$, foi realizado teste ergoespirométrico em esteira rolante (modelo QMCTM 90), a partir de teste contínuo escalonado, com inclinação de $1 \%$, velocidade inicial de $4,5 \mathrm{~km} / \mathrm{h}$ e aumento de $0,5 \mathrm{~km}$ a cada minuto até a exaustão voluntária ou quando mais que um dos seguintes critérios fossem atingidos: aumento no $\mathrm{VO}_{2}$ menor que $2 \mathrm{ml} \cdot \mathrm{kg}^{-1} \cdot \mathrm{min}^{-1}$ para o aumento na intensidade de exercício (platô); razão das trocas respiratórias maior que 1,1 ; frequência cardíaca máxima prevista para a idade fosse atingida (12). Para determinar a potência aeróbia máxima dos indivíduos, foi utilizada a classificação proposta pela American Heart Association (13).

Para avaliação da TMR, os indivíduos estavam em jejum de 12 horas, sem exercícios físicos nas 24 horas prévias à avaliação, e permaneceram em decúbito dorsal durante 10 minutos pré-teste. Após esse período, a avaliação foi realizada durante 30 minutos. $\mathrm{O}$ volume de oxigênio consumido $\left(\mathrm{VO}_{2}\right)$ e o volume de gás carbônico produzido $\left(\mathrm{VCO}_{2}\right)$ foram analisados por calorimetria indireta. A partir desses valores foi calculada a taxa metabólica de repouso mediante a seguinte fórmula: $\mathrm{TMR}=\left(3,9 \times \mathrm{VO}_{2}(\mathrm{~L} / \mathrm{min})\right)+\left(1,1 \times \mathrm{VCO}_{2}(\mathrm{~L} /\right.$ min)) x 1440 (14). Para a quantificação do tipo de substrato oxidado em repouso e durante o exercício foi realizado o quociente respiratório $(\mathrm{QR})$ expresso pela razão $\mathrm{VCO}_{2} / \mathrm{VO}_{2}(15)$. Uma hora antes da realização do exercício, os indivíduos fizeram a refeição padrão descrita anteriormente.

Todos os parâmetros respiratórios foram medidos continuamente em sistema de circuito aberto (modelo QMCTM 90 Metabolic Cart, Quinton , Bothell, USA) e a temperatura ambiente e a umidade relativa do ar foram mantidas entre $21^{\circ} \mathrm{C}$ e $23^{\circ} \mathrm{C}$ e $40 \%$ e $60 \%$, respectivamente.

\section{Análise estatística}

Foi analisada a distribuição das variáveis por meio dos testes Shapiro-Wilk e Kolmogorov \& Smirnov, e a análise de homocedasticidade das variâncias por meio do teste de Levene. Para a comparação dos dois grupos, nos dois momentos do estudo, foi realizado o teste $t$ dependente para as variáveis paramétricas e Mann-Whitney U Test para as não paramétricas. Para o estudo das variações entre os momentos inicial e final $(\Delta)$, considerou-se o teste não paramétrico de Mann-Whitney. Para a comparação entre os grupos em diferentes momentos, foi realizada análise de variância por dois fatores (ANOVA two way) e o teste post hoc de Scheffé para localizar o momento em que a diferença ocorreu. Os resultados foram processados em software STATISTICA for Windows (version 6.0, Statsoft, Tulsa, USA) e estão 
expressos sob a forma de média \pm desvio-padrão (DP) ou mediana \pm semiamplitude interquartílica. O nível de significância adotado para as análises foi o de $5 \%$.

\section{RESULTADOS}

Dos 30 indivíduos que iniciaram o estudo, nove foram excluídos por não cumprirem satisfatoriamente $\mathrm{o}$ protocolo de exercício (frequência mínima de $3 \mathrm{x} / \mathrm{se}$ mana) e a suplementação de L-carnitina. Em relação à suplementação, uma mulher relatou efeitos indesejáveis como dores de cabeça e inchaço nas extremidades, sendo a suplementação interrompida. Duas mulheres desistiram do programa por problemas articulares. O grupo suplementado com L-carnitina foi composto por 5 homens e 6 mulheres, enquanto o grupo placebo foi composto por 4 homens e 6 mulheres.

A tabela 1 mostra que ambos os grupos apresentaram excesso de peso, sendo GS classificado como excesso de peso e GP como obeso classe I, de acordo com os critérios da Organização Mundial da Saúde (OMS) para o IMC (7), porém sem diferença significativa entre os valores médios. Os grupos não se diferenciaram na circunferência de abdômen, na AGL em jejum, na TMR, na ingestão energética, no QR em repouso e durante a prática de exercício físico. Os valores de $\mathrm{VO}_{2 \text { máx }}$, em ambos os grupos, encontram-se abaixo do esperado para a idade, sendo estes classificados como baixa condição, segundo os critérios da American Heart Association (13).

Assim, os grupos foram considerados homogêneos no momento inicial por não apresentarem diferenças significativas $(\mathrm{p}>0,05)$ nos valores médios em nenhuma das variáveis estudadas (Tabela 1 ).

Após o período experimental, não houve variação significativa $(\mathrm{p}>0,05)$ nos valores de IMC, e os dois grupos permaneceram na mesma classificação (Tabela 2). O percentual de gordura corporal e a circunferência de abdômen também não apresentaram alterações significativas $(\mathrm{p}>0,05)$ entre os grupos nos diferentes momentos. Em relação à ingestão energética, os grupos mantiveram o mesmo consumo de calorias do momento inicial. Além disso, a variação mediana $(\Delta)$ entre os momentos não diferiu entre os grupos para todas as variáveis citadas (Tabela 2 ).

As concentrações de AGL em jejum foram semelhantes $(\mathrm{p}>0,05)$ em ambos os grupos nos diferentes momentos. Ao analisar as variações medianas dos grupos, foi observada diferença significativa (Tabela 3 ).
As concentrações de AGL, no pré e pós-exercício, não foram estatisticamente diferentes após a suplementação em GS e GP, e as respectivas variações também não diferiram $(\mathrm{p}<0,05)$ entre os grupos (Tabela 3$)$.

Tabela 1. Comparação entre os grupos suplementados com L-carnitina e placebo de acordo com a idade, peso, estatura, índice de massa corpórea (IMC), circunferência abdominal (CAbd), porcentagem de gordura corporal, ingestão calórica, ácidos graxos livres (AGL) em jejum, pré e pós-exercício, taxa metabólica de repouso (TMB), quociente respiratório (QR) e potência aeróbia máxima $\left(\mathrm{VO}_{2 \text { máx }}\right)$

\begin{tabular}{|c|c|c|c|}
\hline Variáveis & $\begin{array}{c}\text { L-carnitina } \\
(\mathrm{n}=11)\end{array}$ & $\begin{array}{l}\text { Placebo } \\
(n=10)\end{array}$ & ${ }^{\star} p$ \\
\hline Idade (anos) & $46,72 \pm 4,76$ & $44,70 \pm 4,99$ & 0,353 \\
\hline Peso (kg) & $82,35 \pm 16,36$ & $83,57 \pm 14,35$ & 0,859 \\
\hline Estatura (cm) & $169,82 \pm 10,39$ & $164,75 \pm 7,43$ & 0,218 \\
\hline IMC (kg/m²) & $28,37 \pm 3,38$ & $30,68 \pm 4,22$ & 0,180 \\
\hline CAbd (cm) & $99,13 \pm 10,02$ & $98,95 \pm 10,96$ & 0,968 \\
\hline Gordura corporal (\%) & $34,94 \pm 6,07$ & $37,16 \pm 6,99$ & 0,445 \\
\hline Ingestão calórica (kcal/dia) & $2422,2 \pm 734,1$ & $2298,7 \pm 599,3$ & 0,710 \\
\hline AGL jejum (mmol/L)+ & $0,73 \pm 0,13$ & $0,52 \pm 0,09$ & 0,204 \\
\hline AGL pré-exercício (mmol/L)+ & $0,47 \pm 0,16$ & $0,45 \pm 0,19$ & 0,593 \\
\hline AGL pós-exercício (mmol/L)+ & $0,64 \pm 0,32$ & $0,57 \pm 0,21$ & 0,171 \\
\hline TMR (kcal/dia) & $1609,29 \pm 341,98$ & $1523,28 \pm 277,62$ & 0,537 \\
\hline QR repouso & $0,83 \pm 0,08$ & $0,85 \pm 0,12$ & 0,253 \\
\hline QR exercício (30 minutos) & $0,94 \pm 0,08$ & $0,94 \pm 0,06$ & 0,674 \\
\hline $\mathrm{VO}_{2 \text { máx }}\left(\mathrm{ml} \cdot \mathrm{kg}^{-1} \cdot \mathrm{min}^{-1}\right)$ & $26,01 \pm 3,41$ & $22,73 \pm 5,00$ & 0,092 \\
\hline
\end{tabular}

Dados expressos em média \pm desvio-padrão ou mediana \pm semiamplitude interquartílica $\left(^{+}\right)$. * Diferenças entre grupos: test $t$ Student ou Mann-Whitney U Test $\left(^{+}\right)$.

Tabela 2. Efeito da suplementação de L-carnitina sobre o índice de massa corpórea (IMC), circunferência abdominal, porcentagem de gordura corporal e ingestão calórica em indivíduos com excesso de peso, previamente treinados

\begin{tabular}{|c|c|c|c|}
\hline \multirow{2}{*}{ Variáveis } & \multicolumn{2}{|c|}{ Momentos do estudo } & \multirow{2}{*}{$\Delta$} \\
\hline & Inicial (M 0) & Final (M 1) & \\
\hline \multicolumn{4}{|l|}{ IMC $\left(\mathrm{kg} / \mathrm{m}^{2}\right)$} \\
\hline L-carnitina (G1) & $28,37 \pm 3,38^{a}$ & $28,38 \pm 3,50^{\mathrm{a}}$ & $-0,07$ \\
\hline Placebo (G2) & $30,68 \pm 4,22^{\mathrm{a}}$ & $30,54 \pm 4,21^{\text {a }}$ & $-0,17$ \\
\hline \multicolumn{4}{|c|}{ Circunferência abdominal (cm) } \\
\hline L-carnitina (G1) & $99,13 \pm 10,02^{\mathrm{a}}$ & $98,68 \pm 9,56^{\mathrm{a}}$ & 0,50 \\
\hline Placebo (G2) & $98,95 \pm 10,96^{a}$ & $98,25 \pm 11,05^{\mathrm{a}}$ & $-0,25$ \\
\hline \multicolumn{4}{|l|}{$\%$ Gordura corporal } \\
\hline L-carnitina (G1) & $34,94 \pm 6,07^{\mathrm{a}}$ & $34,77 \pm 5,77^{\mathrm{a}}$ & 0,27 \\
\hline Placebo (G2) & $37,16 \pm 6,99^{\mathrm{a}}$ & $36,60 \pm 7,14^{a}$ & $-0,66$ \\
\hline \multicolumn{4}{|c|}{ Ingestão energética (kcal/dia)+ } \\
\hline L-carnitina (G1) & $2422,2 \pm 734,1^{\mathrm{a}}$ & $2170,3 \pm 599,1^{\mathrm{a}}$ & $-244,66$ \\
\hline Placebo (G2) & $2298,7 \pm 599,3^{a}$ & $2157,6 \pm 707,8^{\mathrm{a}}$ & $-126,00$ \\
\hline
\end{tabular}

Dados expressos em média + desvio-padrão ou mediana + semiamplitude interquartílica (+). Asterisco $\left(^{*}\right)$ e letras diferentes indicam diferença significativa $(p<0,05)$. Diferenças entre grupos e em diferentes momentos análise de variância por dois fatores (ANOVA two way) e teste post hoc de Scheffé. Diferenças entre as variações expressas em mediana (M1-M0; $\Delta$ ) teste não paramétrico de Mann-Whitney. 
Tabela 3. Efeito da suplementação de L-carnitina sobre as concentrações de ácido graxo livre (AGL), taxa metabólica de repouso (TMR), quociente respiratório $(\mathrm{QR})$ e o consumo máximo de oxigênio $\left(\mathrm{VO}_{2 \text { máx }}\right)$ em indivíduos com excesso de peso, previamente treinados

\begin{tabular}{|c|c|c|c|}
\hline \multirow{2}{*}{ Variáveis } & \multicolumn{2}{|c|}{ Momentos do estudo } & \multirow{2}{*}{$\Delta$} \\
\hline & Inicial (M 0) & Final (M 1) & \\
\hline \multicolumn{4}{|c|}{ AGL em jejum (mmol/L)+ } \\
\hline L-carnitina (G1) & $0,65 \pm 0,20^{\mathrm{a}}$ & $0,62 \pm 0,22^{\mathrm{a}}$ & $-0,15$ \\
\hline Placebo (G2) & $0,54 \pm 0,15^{\mathrm{a}}$ & $0,69 \pm 0,19^{a}$ & $0,27^{*}$ \\
\hline \multicolumn{4}{|c|}{ AGL pré-exercício $(\mathrm{mmol} / \mathrm{L})^{+}$} \\
\hline L-carnitina (G1) & $0,38 \pm 0,38^{a}$ & $0,40 \pm 0,30^{\mathrm{a}}$ & 0,25 \\
\hline Placebo (G2) & $0,53 \pm 0,66^{\mathrm{a}}$ & $0,48 \pm 0,51^{\mathrm{a}}$ & 0,22 \\
\hline \multicolumn{4}{|c|}{ AGL pós-exercício $(\mathrm{mmol} / \mathrm{L})^{+}$} \\
\hline L-carnitina (G1) & $0,63 \pm 0,95^{a}$ & $0,57 \pm 0,45^{\mathrm{a}}$ & 0,17 \\
\hline Placebo (G2) & $0,74 \pm 0,69^{\mathrm{a}}$ & $0,85 \pm 0,61^{\mathrm{a}}$ & 0,27 \\
\hline \multicolumn{4}{|l|}{ TMR (kcal/dia) } \\
\hline L-carnitina (G1) & $1609,29 \pm 341,98^{\mathrm{a}}$ & $1564,32 \pm 442,85^{\mathrm{a}}$ & 0,06 \\
\hline Placebo (G2) & $1523,28 \pm 277,62^{\mathrm{a}}$ & $1629,41 \pm 324,44^{\mathrm{a}}$ & $-0,02$ \\
\hline \multicolumn{4}{|l|}{ QR em repouso } \\
\hline L-carnitina (G1) & $0,83 \pm 0,08^{a}$ & $0,83 \pm 0,10^{\mathrm{a}}$ & 3,69 \\
\hline Placebo (G2) & $0,85 \pm 0,12^{\mathrm{a}}$ & $0,85 \pm 0,05^{\mathrm{a}}$ & $-1,01$ \\
\hline \multicolumn{4}{|c|}{ QR em exercício (30 min) } \\
\hline L-carnitina (G1) & $0,94 \pm 0,08^{a}$ & $0,96 \pm 0,08^{a}$ & 0,01 \\
\hline Placebo (G2) & $0,94 \pm 0,06^{\mathrm{a}}$ & $0,96 \pm 0,07^{a}$ & $-0,01$ \\
\hline \multicolumn{4}{|l|}{$\mathrm{VO}_{2 \max }(\mathrm{mL} / \mathrm{kg} / \mathrm{min})$} \\
\hline L-carnitina (G1) & $26,01 \pm 3,41^{\mathrm{a}}$ & $26,63 \pm 4,61^{\mathrm{a}}$ & 0,50 \\
\hline Placebo (G2) & $22,73 \pm 5,00^{\mathrm{a}}$ & $24,33 \pm 5,48^{\mathrm{a}}$ & 1,25 \\
\hline
\end{tabular}

Dados expressos em média + desvio-padrão ou mediana + semiamplitude interquartílica $\left({ }^{+}\right)$. Asterisco $\left(^{\star}\right)$ e letras diferentes indicam diferença significativa $(p<0,05)$. Diferenças entre grupos e em diferentes momentos análise de variância por dois fatores (ANOVA two way) e teste post hoc de Scheffé. Diferenças entre as variações expressas em mediana (M1-M0; $\Delta$ ) teste não paramétrico de Mann-Whitney.

Apesar de a taxa metabólica de repouso (TMR) ter sofrido discreta redução em GS e aumento em GP, após o período experimental, esses resultados não foram significativos $(\mathrm{p}>0,05)$ quando comparadas as variações entre os momentos e entre os grupos nos diferentes momentos (Tabela 3 ). Também não foram observadas variações significativas $(\mathrm{p}>0,05)$ em relação ao quociente respiratório, tanto em repouso quanto em exercício, entre os momentos e grupos (Tabela 3). Os valores de $\mathrm{VO}_{2 \text { maxx }}$, expressos na tabela 3 , apresentaram discreto aumento entre os momentos inicial e final, em ambos os grupos, porém sem variações significativas $(\mathrm{p}$ $>0,05)$. Em relação à variação percentual de $\mathrm{VO}_{2 \text { max }}$ dos grupos, não houve diferença significativa (Tabela 3 ).

\section{DISCUSSÃO}

$\mathrm{Na}$ literatura, existem muitas divergências sobre os efeitos da suplementação de L-carnitina na oxidação de substratos energéticos. No presente estudo, não houve mudanças significativas na composição corporal, no quociente respiratório, na TMR e nas AGLs, sugerindo que a taxa metabólica e a oxidação de gorduras não tenham sido influenciadas pela suplementação de L-carnitina. No entanto, no GP foi observado aumento de $63,35 \%$ das concentrações de AGL, que poderia estar relacionado ao discreto aumento do $\mathrm{VO}_{2 \text { máx }}$ $(7,21 \%)$, apesar de não ser significante, mesmo quando comparado ao GS. Segundo Bassett e Howley (16), o treinamento aeróbico causa aumento nas atividades das enzimas mitocondriais, elevando a oxidação de lipídios.

De acordo com Braun e Horton (17), em situações normais de repouso, não existem diferenças no turnover, oxidação de glicose e lipídios e quociente respiratório entre os sexos e nas diferentes fases do ciclo menstrual das mulheres, o que descarta possíveis influências dessas variáveis nos resultados obtidos.

Os achados em relação à suplementação corroboram com os de Natali e cols. (18), que não encontraram diferenças significativas para os níveis circulantes de glicose, lactato, piruvato, ácidos graxos livres, triglicerídios, glicerol e $\beta$-hidroxibutirato no estado de repouso após infusão de L-carnitina ou salina em jovens de ambos os sexos.

Enquanto no estudo de Gorostiaga e cols. (19), com o objetivo de avaliar o aumento da oxidação de ácidos graxos em repouso, tenha encontrado resultados positivos com doses semelhantes à do presente estudo (aproximadamente 2 gramas/dia), outros, utilizando infusão intravenosa de carnitina ( $3 \mathrm{~g})(17)$, doses inferiores e semelhantes (20-22), ou doses superiores ( 4 a $6 \mathrm{~g} /$ dia), não confirmaram tais achados (23-24).

Recentemente, Lee e cols. (25) verificaram por biópsia muscular que a suplementação de L-carnitina ( $4 \mathrm{~g} /$ dia $)$ não alterou a expressão gênica da proteína ligadora de ácido graxo (FABPc), indicadora da capacidade de oxidação de ácidos graxos no músculo, nem a atividade da $\beta$-hidroxiacil CoA desidrogenase.

Em repouso, aproximadamente $70 \%$ dos ácidos graxos liberados na circulação retornam aos triacilgliceróis em vez de serem oxidados. Nesse caso, o aumento da concentração de carnitina na circulação não teria efeitos adicionais sobre a oxidação de gorduras, uma vez que o fator limitante da oxidação não está ligado à disponibilidade de substratos para a oxidação (26).

Além do repouso, a carnitina poderia ser útil como suplemento nas situações em que os lipídios sejam usados predominantemente como fonte energética e este- 
jam disponíveis, como ocorre nos exercícios de baixa a moderada intensidade (27).

No entanto, após as sessões de exercício moderado $\left(60 \%\right.$ do $\left.\mathrm{VO}_{2 \text { max }}\right)$ no presente estudo, os níveis plasmáticos de AGLs aumentaram cerca de $66 \%$ e $25 \%$ acima dos níveis de repouso para GS e 39\% e 77\% para GP nos dois momentos, respectivamente. Embora os aumentos tenham sido maiores para GP, as diferenças não foram significativas entre os momentos para o mesmo grupo, nem entre os grupos suplementados. Após o período experimental, o delta foi menor para o GS, demonstrando que a suplementação de L-carnitina não aumentou a mobilização de gordura do tecido adiposo.

Alterações na oxidação de gorduras e da taxa metabólica de repouso poderiam ser decorrentes de mudanças na composição corporal. Todavia, não houve alteração da composição corporal no presente estudo, corroborando com os achados de outros autores. Villani e cols. (24) observaram que, em mulheres na pré-menopausa com excesso de gordura corporal, a suplementação de L-carnitina (4 g/dia), acompanhada de exercícios físicos de moderada intensidade, durante oito semanas, não alterou significativamente o peso corporal, percentual de gordura e de massa corporal magra das participantes do estudo.

Em contrapartida, Pistone e cols. (28) avaliaram o efeito da suplementação de L-carnitina ( $2 \mathrm{~g} /$ dia $)$ associado à prática de atividade física durante 30 dias sobre a massa muscular e gordura corporal de idosos. Os autores observaram modificações significativas $(\mathrm{p}<0,05)$ na massa gorda e na massa muscular respectivamente $(-3,1 \mathrm{~kg} \mathrm{e}+2,1$ $\mathrm{kg})$ quando comparadas ao placebo $(-0,5 \mathrm{~kg} \mathrm{e}+0,2 \mathrm{~kg})$.

Já em 2005, Broad e cols. (29), em estudo randomizado, duplo-cego, placebo-controlado e crossover, verificaram que a suplementação com 3 gramas por dia de L-carnitina L-tartarato ( 2 g de L-carnitina/dia), durante quatro semanas, não tem efeito sobre oxidação de substratos energéticos ou no desempenho durante o exercício.

É descrito que os níveis teciduais e séricos de carnitina sofrem declínios com o envelhecimento em humanos e animais, em associação à perda de massa muscular. Logo, quanto maior for essa perda, menores serão as concentrações de L-carnitina. Constantin-Teodosiu e cols. (30) demonstraram que as concentrações plasmáticas de carnitina livre correlacionaram-se inversamente com a idade em mulheres.

De maneira oposta e apesar de não estar claramente estabelecido, especula-se que indivíduos obesos apre- sentem maiores concentrações de carnitina em relação àqueles com IMC normal. Harper e cols. (31) observaram, em tecidos obtidos por biópsia de fígado e músculo esquelético, que a concentração de carnitina livre, em obesos mórbidos, era duas vezes maior quando comparada ao grupo controle (IMC $<25 \mathrm{~kg} / \mathrm{m}^{2}$ ). Os autores sugerem que esse aumento do conteúdo de carnitina ocorre predominantemente em situações de alto turnover de ácidos graxos, como, por exemplo, na obesidade.

Uma das hipóteses para explicar a ausência de efeitos positivos sobre a composição corporal do presente estudo, à semelhança do estudo de Villani e cols. (24), seria a presença de concentrações normais musculares de carnitina nos indivíduos participantes. Em ambos os estudos, foram excluídos indivíduos vegetarianos, pois se sabe que a carnitina, além de seus precursores lisina e metionina, está presente em maiores quantidades nos alimentos de origem animal. Todavia, não foram realizadas dosagem sérica ou quantificação muscular de carnitina, o que pode ser uma limitação desse estudo.

Outra questão importante é a relação entre a suplementação de L-carnitina e o tempo necessário para aumentar seus estoques musculares. De acordo com Vukovich e cols. (23), se a suplementação de L-carnitina for capaz de aumentar a concentração intramuscular desta, é possível que a oxidação de lipídios também seja maior. Dessa maneira, um período superior de suplementação poderia aumentar essa oxidação, podendo ser uma limitação desse estudo. Todavia, poucos estudos em humanos ou animais encontraram aumento significativo nas concentrações musculares de carnitina em períodos maiores que quatro semanas (6).

Tornopolsky e cols. (32) submeteram seis homens e seis mulheres treinados à corrida em esteira rolante a $65 \%$ do $\mathrm{VO}_{2 \text { máx }}$ com o objetivo de determinar a influência do gênero sobre a utilização de substratos. Após 30 minutos do teste, as concentrações de AGLs permaneceram constantes, sem diferenças significativas para os sexos, embora o QR das mulheres fosse significativamente menor do que o dos homens. Os autores sugerem que os valores de AGLs permaneceram inalterados em função da grande utilização de TGL intramusculares dos indivíduos treinados e principalmente do sexo feminino.

$\mathrm{Na}$ intensidade de exercício, executada no presente estudo, a participação dos lipídios para o fornecimento de energia foi de $19,3 \%$, observada a partir dos valores de QR $(0,94)$ para G1 e G2 no M0. Após o período experimental, a participação dos lipídios para o forneci- 
mento de energia foi de $12,8 \%$ para ambos os grupos, demonstrando ligeira redução da oxidação de gorduras, embora não significativa. Esses achados confirmam os encontrados por outros autores $(23,33)$.

Segundo Cerreteli e Marconi (34) e Stephens e cols. (5), a suplementação de L-carnitina poderia ter efeitos indiretos sobre $\mathrm{o} \mathrm{VO}_{2 \text { máx }}$, por meio da redução da massa corporal gorda e diretamente pelo aumento da disponibilidade e fluxo de substratos energéticos ao ciclo de Krebs, aumento da atividade de enzimas do ciclo de Krebs como piruvato desidrogenase, carnitina palmitoiltransferase e de enzimas da cadeia transportadora de elétrons. O aumento da atividade dessas enzimas potencializaria o fluxo de substratos energéticos para manutenção do "pool" de CoA livre e produção máxima de ATP e possivelmente da potência aeróbia máxima (expressa pelo $\mathrm{VO}_{2 \operatorname{máx}}$ ).

O estudo realizado por Galloway e Broad (35) mostrou, em 14 homens ativos, que a suplementação de carnitina $(2 \mathrm{~g} /$ dia $)$ aumentou a utilização de gordura e o $\mathrm{VO}_{2 \max }$ após 6 semanas, retornando ao basal após quatro semanas de “wash-out”. Não houve modificação da composição corporal nesse período.

De maneira semelhante, Wyss e cols. (27) avaliaram os efeitos da suplementação de L-carnitina ( $3 \mathrm{~g} /$ dia $)$ durante sete dias sobre o $\mathrm{VO}_{2 \text { máx }}$ em condições de hipóxia e normóxia em 7 homens saudáveis. $\mathrm{O}$ consumo máximo de oxigênio foi menor na hipóxia, mas sem diferenças significativas para os dois tratamentos (carnitina e placebo).

Recentes estudos controlados sugerem que a suplementação de L-carnitina não produz efeitos sobre $o$ $\mathrm{VO}_{2 \text { máx }}(29,36)$. A eficácia da L-carnitina no aumento do $\mathrm{VO}_{2 \text { máx }}$, na maioria das vezes, foi demonstrada apenas por estudos com portadores de doenças cardiovasculares ou renais, os quais apresentavam baixa capacidade funcional (3).

Em suma, verificou-se que a suplementação oral de L-carnitina, por quatro semanas, não promoveu alterações sobre a composição corporal e a oxidação de ácidos graxos livres no estado de repouso e durante a prática de exercício físico aeróbico em indivíduos com excesso de peso e obesidade. Desse modo, conclui-se que a suplementação de L-carnitina, nesse tipo de população, é ineficaz na perda de peso, no gasto energético, no uso diferenciado de substratos energéticos e na potência aeróbia.

Declaração: os autores declaram não haver conflitos de interesse científico neste estudo.

\section{REFERÊNCIAS}

1. Coelho CF, Camargo VR, Ravagnani FC. Consumo de suplementos nutricionais por praticantes de musculação em academia de Campo Grande, MS. Rev Nut Pauta. 2007;15(87):41-6.

2. Cerreteli P, Marconi C. L-Carnitine suplementation in humans. The effects on physical performance. Int J Sports Med. 1990;11(1):1-4.

3. Coelho CF, Mota JF, Bragança E, Burini RC. Clinical uses of L-carnitine supplementation. Rev Nutr. 2005;18(5):651-9.

4. Bonnefont JP, Djouadi F, Prip-Buus C, Gobin S, Munnich A, Bastin J. Carnitine palmitoyltransferases 1 and 2: biochemical, molecular and medical aspects. Mol Aspects Med. 2004;25(5-6):495-520.

5. Stephens FB, Constantin-Teodosiu D, Greenhaff PL. New insights concerning the role of carnitine in the regulation of fuel metabolism in skeletal muscle. J Physiol. 2007;581(2):431-44.

6. Heinonen OJ. Carnitine and physical exercise. Sports Med. 1996;22(2):109-32.

7. World Health Organization (WHO). Diet, nutrition and the prevention of chronic diseases. Geneva: WHO/FAO. Expert consultation on diet, nutrition and prevention of chronic diseases, 2002.

8. Rubin MR, Volek JS, Gómez AL, Ratamess NA, French DN, Sharman MJ, et al. Safety measures of L-carnitine L-tartrate supplementation in heathy men. Strength Cond Res. 2001;15(4):486-90.

9. Executive summary of the third report of the National Cholesterol Education Program (NCEP) expert panel on the detection, evaluation, and treatment of high blood cholesterol in adults (Adult Treatment Panel III). JAMA. 2001;285(19):2486-97.

10. Gray DS, Bray GA, Gemayel N, Kaplan K. Effect of obesity on bioeletrical impedance. Am J Clin Nutr. 1989;50(2):255-60.

11. Novak M. Colorimetric ultramicro method for the determination of free fatty acids. Journ Lipid Res. 1965;6(1):431-3.

12. Balke B, Ware R. An experimental study of Air Force Personnel. U.S. Armed Forces Med J. 1959;10(6):675-88.

13. Marins JCB, Giannichi RS. Avaliação e prescrição de atividade física. Shape: Rio de Janeiro; 1996.

14. Branson RD. The measurement of energy expenditure: instrumental, practical considerations and clinical application. Respir Care. 1990;35(1):640-59.

15. Mcardle WD, Katch Fl, Katch VL. Fisiologia do exercício: energia, nutrição e desempenho humano. 4.ed. Guanabara Koogan: Rio de Janeiro; 1998.

16. Bassett DR Jr, Howley ET. Limiting factors for maximum oxygen uptake and determinants of endurance performance. Med Sci Sports Exerc. 2000;32(1):70-84.

17. Braun B, Horton T. Endocrine regulation of exercise substrate utilization in women compared to men. Exerc Sport Sci Rev. 2001;29(4):149-54.

18. Natali A, Santoro D, Brandi LS, Faraggiana D, Ciociaro D, Pecori N, Buzzigoli G, Ferrannini E. Effects of acute hypercarnitinemia during increased fatty substrate oxidation in man. Metabolism. 1993;42(5):594-600.

19. Gorostiaga EM, Maurer CA, Eclache JP. Decrease in respiratory quotient during exercise following L-carnitine supplementation. Int J Sports Med. 1989;10(3):169-74.

20. Oyono-Enguelle S, Freund H, Ott C, Gartner M, Heitz A, Marbach J, Maccari F, et al. Prolonged submaximal exercise and L-carnitine in humans. Eur J Appl Physiol. 1988;58(1-2):53-61.

21. Siliprandi N, Di Lisa F, Pieralisi G, Ripari P, Maccari F, Menabo R, et al. Metabolic changes induced by maximal exercise in human subjects following L-carnitine administration. Bioch Byophys Acta. 1990;1034(1):17-21.

22. Colombani P, Wenk $C$, Kunz I, Krähenbühl S, Kuhnt M, Arnold $M$, et al. Effects of L-carnitine supplementation on physical performance and energy metabolism of endurance-trained ath- 
letes: a double-blind crossover field study. Eur J Appl Physiol. 1996;73(5):434-9.

23. Vukovich MD, Costill DL, Fink WJ. Carnitine supplementation: effect on muscle carnitine and glycogen content during exercise. Med Sci Sports Exerc. 1994;26(9):1122-9.

24. Villani R, Gannon J, Self M, Rich PA. L-carnitine supplementation combined with aerobic training does not promote weight loss in moderately obese women. Int J Sport Nutr. 2000;10(2):199-207.

25. Lee JK, Lee JS, Park H, Cha YS, Yoon CS, Kim CK. Effect of L-carnitine supplementation and aerobic training on FABPc content and $\beta$-HAD activity in human skeletal muscle. Eur J Appl Physiol. 2007;99(2):193-9.

26. Evans AM, Fornasini G. Pharmacokinetics of L-carnitine. Clin Pharmacokinet. 2003;42(11):941-67.

27. Wyss V, Ganzit GP, Rienzi A. Effects of L-carnitine administration on $\mathrm{VO}_{2 \max }$ and the aerobic-anaerobic threshold in normoxia and acute hypoxia. Eur J Appl Physiol. 1990;60(1):1-6.

28. Pistone G, Marino AD, Leotta C, Dell'Arte S, Finocchiaro G, Malaguarnera $M$. Levocarnitine administration in elderly subjects with rapid muscle fatigue. Drugs aging 2003;20(10):761-7.

29. Broad EM, Maughan RJ, Galloway SDR. Effects of four weeks Lcarnitine L-tartrate ingestion on substrate utilization during prolonged exercise. IJSNEM. 2005;15(6):665-79.
30. Constantin-Teodosiu D, Young S, Wellock F, Short AH, Burden RP, Morgan AG, et al. Gender and age differences in plasma carnitine, muscle strength and exercise tolerance in hemodialysis patients. Nephrol Dial Transplant. 2002;17(10):1808-13.

31. Harper P, Wadstrom C, Backman L, Cederblad G. Increased liver carnitine content in obese women. Am J Clin Nutr. 1995;61(1): 18-25.

32. Tornopolsky LJ, MacDougall JD, Atkinson SA, Tarnopolsky MA, Sutton JR. Gender differences in substrate for endurance exercise. J Appl Physiol. 1990;68(1):302-8.

33. Greig C, Finch KM, Jones DA, Cooper M, Sargeant AJ, Forte CA. The effect of oral supplementation with L-carnitine on maximum and submaximum exercise capacity. Eur J Appl Physiol. 1987;56(4):457-60.

34. Cerreteli P, Marconi C. L-Carnitine supplementation in humans. The effects on physical performance. Int J Sports Med. 1990;11(1):1-4.

35. Galloway SDR, Broad EM. Effects of L-carnitine L-tartrate supplementation on substrate metabolism during exercise. In: Experimental Biology, April, 2004. Washington DC, 2004. p. A770.

36. Stuessi $C$, Hofer $P$, Meier C. L-carnitine and the recovery from exhaustive endurance exercise: a randomized, double-blind, placebo-controlled trial. Eur J Appl Physiol. 2005;95(5-6):431-5. 